\title{
Quantum Optimal Control for Bose-Einstein-Condensates at Complex Space
}

\author{
Quan-Fang Wang \\ ${ }^{1}$ Chinese University of Hong Kong, Shatin, N.T.,Hong Kong \\ E-mail: quanfangwang@hotmail.com
}

\begin{abstract}
Quantum control of Bose-Einstein-Condensates is interesting topic in the areas of control and physics. In this work, Gross-Pitaevskii equation expressed BoseEinstein-Condensates is considered as control target. Full theoretical proof for the existence of quantum optimal control is provided for cubical Schrödinger equation in complex Hilbert spaces.
\end{abstract}

PACS numbers: 02.30.Yy,03.75.Gg,33.20.Tp

Keywords: Quantum control, Bose-Einstein-Condensates, Complex Hilbert space, Variational method, Eular-Lagrange system.

Submitted to: J. Phys. A: Math. Gen. 


\section{Introduction}

Bose-Einstein-Condensates (BEC) theory is proposed by Bose and Einstein at 1922, one dimension bosonic atoms can be collapsed to condensate for $10^{3}$ particles in lab at JILA 1995 of last century; Nobel Prize 2001. After that, experimental and theoretical investigation sufficiently reported in literatures for $2 \mathrm{D}$ and $3 \mathrm{D}$ dimension (cf. [2], [5]). Atomic condensates ${ }^{87} \mathrm{Rb},{ }^{7} \mathrm{Li},{ }^{23} \mathrm{Na},{ }^{52} \mathrm{Cr},{ }^{39} \mathrm{~K}$ had been made in laboratory. BEC topic had been spread to a various fields: mathematics, chemistry, and so forth. At the viewpoint of control, BEC is seldom regarded as control purpose. Physically, control of a condensate could be occurred at experiment methodology, experimentation result is already appeared [2].

Theoretic control theory is delay for Bose-Einstein-Condensate. For nonlinear Gross-Pitaevskii (G-P) equation, control can be forced at condensate creation consisting of two external factors. One is the electromagnetic field, usual in the quadratic form of spatial variable, another is optical potential, trapped laser for ultracold particles, is in the form of time independed shaped pulse, wave function. To existing physical BEC experiments, a few question is arising in here: it is necessary to apply control theory to a condensates; it is useful for creating a condensate; control can make sense in practice. Absolutely. Qualitatively experimental result for BEC status of a particle could not as access for others. Theoretical survey of quantitatively controlling is indicating adjustment of magnetic trap minimum, and optical confinement. Quantum control for Bose-Einstein-Condensate can be found for control of external force at Hamiltonian feedback controller, numerical control of magnetic field.

In this paper, quantum optimal control is interesting in the theoretical aspect for Bose-Einstein-Condensates, a description of Gross-Pitaevskii equation, in complex space systematically. Taking G-P equation in the framework of variational method in the complex Hilbert space; applying control theory to quantum system of G-P equation to BEC; supplying full proof for the existence quantum optimal control. Finally, theoretical support to find substantial control of bosons from ground state to condensate status.

Divided this paper into a couple of sections. Section 2 is physical background of Bose-Einstein-Condensates. Section 3 is to do mathematical setting for BEC in complex

Hilbert spaces. Section 4 is quantum control theory for BEC using the framework of variational method. Section 5 concludes current work.

\section{Physical background}

In physical field, as an ultra-cold vapor of bosonic atoms are trapped in electro-magnetic well, one can create pure condensates as they are cooling to the temperature than a BEC criticalness. As that taking place, a BEC is located into a optical lattices, a molasses surrounding the particles. Quantum system for ultracold atoms is unique wellposed for experimental controlling. Suppose $\mathbf{n}$ number particles are in a condensates at optical lattice, each particle as $P_{n}$ for $n=1,2, \cdots, \mathbf{n}$. Set $\mathbf{x}=\left(x_{1}, x_{2}, x_{3}\right)$ in $\mathbf{R}^{3}$. 
Bose-Einstein-Condensates can be usually taken as Gross-Pitaevskii (G-P) equation, a cubically nonlinear Schrödinger equation (cf. [4])

$$
\mathrm{i} \hbar \frac{\partial \psi_{n}}{\partial t}=-\frac{\hbar^{2}}{2 M} \Delta \psi_{n}+u_{n}(\mathbf{x}) \psi_{n}+\mathbf{n} \nu\left|\psi_{n}\right|^{2} \psi_{n}
$$

for $n=1,2, \cdots, \mathbf{n}$. In here, $\hbar$ is Planck constant, $M$ is atomic mass, $\psi_{n}(t, \mathbf{x})$ is condensate wave function of $n$-th particle $P_{n}$ at BEC, $\mathbf{n}$ is total number of atoms in the condensate, and $\nu=4 \pi \hbar^{2} a / M$ is interacting constant, $a \in \mathbf{R}$ is characteristic scatter length. For control purpose, neglect parameters and formulation in magnetic field and optical regime, total external force $V\left(u_{n}\right)$ is unified set $u_{n}(\mathbf{x})=v_{n}^{1}(\mathbf{x})+v_{n}^{2}(\mathbf{x})$, and external potential $v_{n}^{1}(\mathbf{x})$ is electro-magnetic field for experiment BEC of a particle $P_{n}$, in general,

$$
v_{n}^{1}(\mathbf{x})=M \sigma_{0}^{2} \frac{|\mathbf{x}|^{2}}{2} \text { for } \sigma_{0} \in \mathbf{R} .
$$

Optical potential for particle $P_{n}$ in physical experiments (cf. [4]) as

$$
v_{n}^{2}(\mathbf{x})=\mu \sum_{t=1}^{3} \frac{\hbar^{2} \mathbf{x}^{2}}{M} \sin ^{2}\left(x_{t} x_{t}\right), x_{t} \in \mathbf{R},
$$

and $\mu>0$ is a dimensionless parameter for expressing the depth of optical lattice. System (1) is representing an interacting $n$ particles BEC condensate.

In practice, although Bose-Einstein-Condensates is taking as Gross-Pitaevskii equation in the form of cubically complex Schrödinger equation, the task in this work is to discuss control theory in complex functional analysis space to ensure control solution is making sense in real space, and meaningful in realistic world. That is, complex G-P equation (1) is formula reflection of a real BEC condensate.

\section{Variational method framework}

Despite it is real particles of bosonic atoms in a condensate, to test and check control theory is worked for BEC condensate. Consider Bose-Einstein-Condensate in the form of Gross-Pitaevskii equation (1), to do mathematically setting in complex Hilbert spaces. Suppose $\Omega$ is an open bounded set of $\mathbf{R}^{3}$ and $Q=(0, T) \times \Omega$ for $T>0,(t, \mathbf{x}) \in Q$. In G-P system (1), $u_{n}(\mathbf{x})$ is control input of particle $P_{n}$. Control $u_{n}(\mathbf{x})$ is depended on

spatial variable $\mathbf{x}$, and independent of time $t$. For complex-valued function $\psi_{n}$, define complex solution space $\mathbb{L}^{2}(\Omega)$ and $\mathbb{H}_{0}^{1}(\Omega)$ corresponding to $L^{2}(\Omega)$ and $H_{0}^{1}(\Omega)$, use the notations $\mathbb{H}=\mathbb{L}^{2}(\Omega), \mathbb{V}=\mathbb{H}_{0}^{1}(\Omega)$ for two complex Hilbert spaces. Further, $\mathbb{V}^{\prime}$ is complex conjugate space of $\mathbb{V}$. Actually, $(\mathbb{V}, \mathbb{H})$ is a complex Gelfand triple spaces $\mathbb{V} \hookrightarrow \mathbb{H} \hookrightarrow \mathbb{V}^{\prime}$, two embeddings are continuous, dense and compact. For $\psi_{n}=\psi_{n}^{1}+\mathbf{i} \psi_{n}^{2} \in \mathbb{L}^{2}(\Omega)$, $\psi_{n}^{1}, \psi_{n}^{2} \in L^{2}(\Omega)$, define norm of $\psi_{n}$ in complex space $\mathbb{L}^{2}(\Omega)$ as

$$
\left\|\psi_{n}\right\|_{\mathbb{L}^{2}(\Omega)}=\left(\left\|\psi_{n}^{1}\right\|_{L^{2}(\Omega)}^{2}+\left\|\psi_{n}^{2}\right\|_{L^{2}(\Omega)}^{2}\right)^{\frac{1}{2}} .
$$

If $\boldsymbol{\psi}^{n}=\boldsymbol{\psi}_{n}^{1}+\mathbf{i} \psi_{n}^{2} \in \mathbb{L}^{2}(\Omega)$, then inner product of $\psi_{n}$ and $\boldsymbol{\psi}_{n}$ in complex space $\mathbb{L}^{2}(\Omega)$ can be defined by

$$
\left.\left(\psi_{n}, \boldsymbol{\psi}_{n}\right)_{\mathbb{L}^{2}(\Omega)}=\left(\left(\psi_{n}^{1}, \boldsymbol{\psi}_{n}^{1}\right)_{L^{2}(\Omega)}+\left(\psi_{n}^{2}, \boldsymbol{\psi}_{n}^{2}\right)\right)_{L^{2}(\Omega)}\right)+\mathbf{i}\left(\left(\psi_{n}^{2}, \boldsymbol{\psi}_{n}^{1}\right)_{L^{2}(\Omega)}-\left(\psi_{n}^{1}, \boldsymbol{\psi}_{n}^{2}\right)_{L^{2}(\Omega)}\right) .
$$


For $\psi_{n} \in \mathbb{H}_{0}^{1}(\Omega)$, the norm of $\psi_{n}$ in complex space $\mathbb{H}_{0}^{1}(\Omega)$ is

$$
\left\|\psi_{n}\right\|_{\mathbb{H}_{0}^{1}(\Omega)}=\left(\left\|\psi_{n}^{1}\right\|_{H_{0}^{1}(\Omega)}^{2}+\left\|\psi_{n}^{2}\right\|_{H_{0}^{1}(\Omega)}^{2}\right)^{\frac{1}{2}} .
$$

If $\boldsymbol{\psi}_{n} \in \mathbb{H}_{0}^{1}(\Omega)$, then inner product of $\psi_{n}$ and $\boldsymbol{\psi}_{n}$ in complex space $\mathbb{H}_{0}^{1}(\Omega)$ is defined as

$$
\left(\psi_{n}, \boldsymbol{\psi}_{n}\right)_{\mathbb{H}_{0}^{1}(\Omega)}=\left(\psi_{n}^{1}, \boldsymbol{\psi}_{n}^{1}\right)_{H_{0}^{1}(\Omega)}+\left(\psi_{n}^{2}, \boldsymbol{\psi}_{n}^{2}\right)_{H_{0}^{1}(\Omega)} .
$$

Definition 1 Complex space $W(0, T)$ is said as solution space of $\psi_{n}$

$$
W(0, T)=\left\{\psi_{n} \mid \psi_{n} \in L^{2}(0, T ; \mathbb{V}), \psi_{n}^{\prime} \in L^{2}\left(0, T ; \mathbb{V}^{\prime}\right)\right\} .
$$

The norm of $\psi_{n}$ in complex space $W(0, T)$ can be defined as

$$
\left\|\psi_{n}\right\|_{W(0, T)}=\left(\left\|\psi_{n}\right\|_{L^{2}(0, T ; \mathbb{V})}^{2}+\left\|\psi_{n}^{\prime}\right\|_{L^{2}\left(0, T ; \mathbb{V}^{\prime}\right)}^{2}\right)^{\frac{1}{2}} .
$$

If $\psi_{n}, \boldsymbol{\psi}_{n} \in W(0, T)$, then definition of inner product is

$$
\left(\psi_{n}, \boldsymbol{\psi}_{n}\right)=\left(\psi_{n}, \boldsymbol{\psi}_{n}\right)_{\mathbb{V}}+\left(\psi_{n}^{\prime}, \boldsymbol{\psi}_{n}^{\prime}\right)_{\mathbb{V}^{\prime}}
$$

Thus, $W(0, T)$ is a complex Hilbert space equipped by above norm and inner product.

Definition 2 A function $\psi_{n}$ is said as weak solution of BEC system in the form of $G-P$ equation (1) for $n$-th particle $P_{n}, n=1,2, \cdots, \mathbf{n}$, if $\psi_{n} \in W(0, T)$ and satisfy

$$
\begin{aligned}
& \int_{0}^{T} \int_{\Omega} \mathbf{i} \hbar \frac{\partial \psi_{n}}{\partial t} \mathrm{n}^{n} d \mathbf{x} d t \\
= & \frac{\hbar^{2}}{2 M} \int_{0}^{T} \int_{\Omega} \psi_{n \mathbf{x}} \mathrm{n}_{\mathbf{x}}^{n} d \mathbf{x} d t+\int_{0}^{T} \int_{\Omega} u_{n}(\mathbf{x}) \psi_{n} \mathrm{n}^{n} d \mathbf{x} d t+\mathbf{n} \nu \int_{0}^{T} \int_{\Omega}\left|\psi_{n}\right|^{2} \psi_{n} \mathrm{n}^{n} d \mathbf{x} d t,
\end{aligned}
$$

where $\mathrm{n}^{n}$ is a arbitrary test function by the means of distribution in space $\mathcal{D}^{\prime}(0, T)$, $\mathrm{n}^{n} \in C^{1}(0, T ; \mathbb{V})$ and $\mathrm{n}^{n}(T)=0$.

Citing [3, 9], from weak form (2) to get existence theorem of weak solution.

Theorem 1 For $\psi_{n 0} \in \mathbb{V}$ of particle $P_{n}, n=1,2, \cdots, \mathbf{n}$, then there exists a unique solution $\psi_{n} \in W(0, T)$ for $G-P$ equation (1).

Proof. As in [3], using Faedo-Galerkin method to construct an approximate solution for BEC system (1). From Gelfand triple spaces, $\mathbb{V} \hookrightarrow \mathbb{H}$ is compact, then there exists an orthogonal basis of $\mathbb{H},\left\{w^{j}\right\}_{j=1}^{\infty}$ consisting of eigenfunctions of $A=\Delta$, such that $A w^{j}=\lambda^{j} w^{j}$ for all $j, 0<\lambda^{1} \leq \lambda^{2} \leq, \cdots, \lambda^{j} \rightarrow \infty$ as $j \rightarrow \infty$. $G^{i}$ is the orthogonal projection of $\mathbb{H}$ (or $\mathbb{V}$ ) onto the space spanned by $\left\{w^{1}, \cdots, w^{i}\right\}$. For each $i \in N$, an approximate solution is defined for G-P equation (1) by $\psi_{n}^{i}(t)=\sum_{j=1}^{i} a_{n}^{i j}(t) w_{j}$, $n=1,2, \cdots, \mathbf{n}$, and $a_{n}^{i j}(t)$ is real-valued coefficient function. Taking $\mathrm{n}^{n}=w^{j}$ at weak form $(2)$, then approximate solution $\psi_{n}^{i}(t)$ satisfy ordinary differential equation $(1 \leq i \leq N)$ as

$$
\left\{\begin{array}{l}
\int_{0}^{T} \int_{\Omega} \mathbf{i} \hbar \frac{\partial \psi_{n}^{i}}{\partial t} w^{j} d \mathbf{x} d t \\
=\frac{\hbar^{2}}{2 M} \int_{0}^{T} \int_{\Omega} \nabla \psi_{n}^{i} \nabla w^{j} d \mathbf{x} d t+\int_{0}^{T} \int_{\Omega} u_{n}(\mathbf{x}) \psi_{n}^{i} w^{j} d \mathbf{x} d t+\mathbf{n} \nu \int_{0}^{T} \int_{\Omega}\left|\psi_{n}^{i}\right|^{2} \psi_{n}^{i} w^{j} d \mathbf{x} d t \\
\psi_{n}^{i}(0)=\psi_{n 0}^{i},
\end{array}\right.
$$


Substitute $\psi_{n}^{i}$ as approximate form in (3), from standard theory of ODE, ensure a unique local solution $\left\{\psi_{n}^{i}\right\}, i=1,2, \cdots, N$ for approximate system (3). For $\psi_{n 0}^{i}$, there exists a $\psi_{n 0} \in \mathbb{V}$ such that

$$
\psi_{n 0}^{i} \rightarrow \psi_{n 0} \quad \text { strongly in } \mathbb{H}_{0}^{1}(\Omega) .
$$

Take analogous argument for $\left\{\psi_{n}^{i}\right\}$, there is a function $\psi_{n} \in \mathbb{V}$, that $\left\|\psi_{n}^{i}-\psi_{n}\right\|_{\mathbb{V}} \rightarrow 0$. Approximate solution $\psi_{n}^{i}$ is bounded in $L^{\infty}(0, T ; \mathbb{V})$. Suppose that $\left\{\psi_{n}^{i}\right\}$ and $\left\{\psi_{n}^{k}\right\}$ are two solutions of G-P equation (1) for $\psi_{n 0}^{i}$ and $\psi_{n 0}^{k}$. From weak form (2) to get

$$
\begin{aligned}
& \frac{\mathbf{i} \hbar}{2} \frac{d}{d t} I^{i k}(t)+\frac{\hbar^{2}}{2 M}\left\|\psi_{n}^{i}-\psi_{n}^{k}\right\|_{\mathbb{V}}^{2} \\
\leq & C(t)\left(\left\|\psi_{n}^{i}-\psi_{n}^{k}\right\|_{\mathbb{H}}^{2}+\mathbf{n} \nu\left\|\left|\psi_{n}^{i}\right|^{2} \psi_{n}^{i}-\left|\psi_{n}^{k}\right|^{2} \psi_{n}^{k}\right\|_{\mathbb{H}}^{2}\right) .
\end{aligned}
$$

In here, $C(t)=\max \left\{u_{n}(\mathbf{x}), 2 \mathbf{n} \nu\left(\left|\psi_{n}^{i}\right|^{2}+\left|\psi_{n}^{k}\right|^{2}\right)\right\}$ and $I^{i k}(t)=\left\|\psi_{n}^{i}(t)-\psi_{n}^{k}(t)\right\|_{\mathbb{H}}^{2}+\| \psi_{n}^{i}(t)-$ $\psi_{n}^{k}(t) \|_{\mathbb{V}}^{2}$. Set $\lambda=\max \left\{\frac{\hbar}{2}, \frac{\hbar^{2}}{2 M}\right\}$ and $C^{\prime}(t)=C(t) / \lambda$ that

$$
\frac{d}{d t} I^{i k}(t) \leq C^{\prime}(t) I^{i k}(t)
$$

Set $I^{i k}(0)=\left\|\psi_{n 0}^{i}-\psi_{n 0}^{k}\right\|_{\mathbb{H}}^{2}+\left\|\psi_{n 0}^{i}-\psi_{n 0}^{k}\right\|_{\mathbb{V}}^{2}$, from Gronwall inequality that

$$
I^{i k}(t) \leq \exp \left(\int_{0}^{T} C^{\prime}(t) d t\right) I^{i k}(0)
$$

It implied $\psi_{n}^{i} \rightarrow \psi_{n}^{k}$ as $i, k \rightarrow \infty$. Taking $\psi_{n}^{k}=\psi_{n}$ to find that $\psi_{n}^{i} \rightarrow \psi_{n}$ in $C(0, T ; \mathbb{H})$. Thus, $C(0, T ; \mathbb{H}) \subset W(0, T)$ to sure Theorem 1 .

\section{Applying quantum control theory}

In complex Hilbert space, consider theoretical existence of quantum optimal control for Bose-Einstein-Condensates. Suppose $\mathcal{U}=L^{2}(\Omega)$ is the space of controls $u_{n}$, and $\mathcal{U}_{a d}$ is a admissible set of $\mathcal{U}$. Mathematically set control $\boldsymbol{u}=\left(v^{1}, \cdots, u^{n}, \cdots, v^{\mathbf{n} *}\right)$, and corresponding optimal control $\boldsymbol{u}^{*}=\left(v^{1 *}, \cdots, v^{n *}, \cdots, v^{\mathbf{n} *}\right)$. Set ground state of $n$-th particle $P_{n}$ as $\psi_{n}\left(0, u_{n}\right)=\psi_{n 0}$. Cost function of $n$-th particle for G-P system (1) is in the form of

$$
J\left(u_{n}\right)=\epsilon^{1}\left\|\psi_{n}^{f}\left(u_{n}\right)-\psi_{n}^{\text {target }}\left(u_{n}\right)\right\|_{\mathbb{H}}^{2}+\epsilon^{2}\left\|u_{n}\right\|_{\mathcal{U}}^{2} .
$$

In criteria function (4), $u_{n} \in \mathcal{U}_{a d}, \psi_{n}^{\text {target }}\left(u_{n}\right)$ is target state, $\psi_{n}^{f}\left(u_{n}\right)$ is observed final state of particle $P_{n}$ at final time $t_{f} . \epsilon^{i}, i=1,2$ are weighted coefficients for balancing the values of inhere cost and running cost. Total cost function $J(\boldsymbol{u})=\sum_{n=1}^{\mathbf{n}} J\left(u_{n}\right)$ for a condensate of $n$ particles.

Two fundamental problems of quantum optimal control for Bose-EinsteinCondensates:

i). find quantum optimal control $u_{n}^{*}, n=1,2, \cdots, \mathbf{n}$ for G-P system (1).

ii). find Eular-Lagrange system for $u_{n}^{*}$. 
For $n$-th particle $P_{n}, u_{n}^{*}$ is said as quantum optimal control for G-P system (1) to cost function (4).

Theorem 2 For $\psi_{n 0} \in \mathbb{V}$ of $n$-th particle $P_{n}, n=1,2, \cdots, \mathbf{n}$, if $\mathcal{U}_{a d}$ is closed convex (bounded) admissible subset of $\mathcal{U}$, then there exists at least one quantum optimal control $u_{n}^{*}$ of Bose-Einstein-Condensates (1) subject to cost function (4).

Proof. Set $J=\inf _{u_{n} \in \mathcal{U}_{a d}} J\left(u_{n}\right)$, since $\mathcal{U}_{a d}$ is non-empty, there is a sequence $\left\{u_{n}^{k}\right\}$ in $\mathcal{U}_{a d}$ such that $\inf _{u_{n} \in \mathcal{U}_{a d}} J\left(u_{n}\right)=\lim _{k \rightarrow \infty} J\left(u_{n}^{k}\right)=J$. Since $\left\{J\left(u_{n}\right)\right\}$ is bounded in $\mathbf{R}^{+}$, and $\mathcal{U}_{a d}$ is closed and convex (bounded) subset of $\mathcal{U}$, there exist a subsequences $\left\{u_{n}^{k^{\prime}}\right\}$ of $\left\{u_{n}^{k}\right\}$ can be extracted, and a $u_{n}^{*} \in \mathcal{U}_{a d}$, such that

$$
u_{n}^{k^{\prime}} \rightarrow u_{n}^{*} \text { weakly in } \mathcal{U} \text { as } k^{\prime} \rightarrow \infty \text {. }
$$

From the existence theorem 1 of weak solution to get estimate $\left\|\psi_{n}\right\|_{\mathbb{H}}^{2}+\left\|\psi_{n}\right\|_{\mathbb{V}}^{2}$ is bounded for $\psi_{n}$ of $n$-th particle $P_{n}$. For control $u_{n}^{k^{\prime}}$, from boundedness of $\mathcal{U}_{a d}$ that

$$
\psi_{n}\left(u_{n}^{k^{\prime}}\right) \text { is bounded in } L^{2}(0, T ; \mathbb{H}) \cap L^{2}(0, T ; \mathbb{V}) .
$$

Setting $\psi_{n}^{*}=\psi_{n}\left(u_{n}^{*}\right)$, there exist a subsequence $\left\{\psi_{n}\left(u_{n}^{k^{\prime \prime}}\right)\right\}$ of $\left\{\psi_{n}\left(u_{n}^{k^{\prime}}\right)\right\}$ and a $\psi_{n}^{*} \in$ $W(0, T)$ such that

$$
\psi_{n}\left(u^{k^{\prime \prime}}\right) \rightarrow \psi_{n}^{*} \quad \text { weakly in } L^{2}(0, T ; \mathbb{H}) \cap L^{2}(0, T ; \mathbb{V}) .
$$

as $k^{\prime \prime} \rightarrow \infty$. Since the embedding $\mathbb{V} \hookrightarrow \mathbb{H}$ is compact, from Aubin-Lions-Temam theorem (cf. [6]), then there is $\bar{\psi}_{n} \in \mathbb{H}$ that

$$
\psi_{n}\left(u^{k^{\prime \prime}}\right) \rightarrow \bar{\psi}_{n} \quad \text { strongly in } L^{2}(0, T ; \mathbb{H}) \quad k^{\prime \prime} \rightarrow \infty,
$$

and get the convergences for $\psi_{n}$ as

$$
\left\{\begin{array}{l}
\frac{\partial \psi_{n}}{\partial t}\left(u^{k^{\prime \prime}}\right) \rightarrow \frac{\partial \bar{\psi}_{n}}{\partial t} \text { weakly in } L^{2}\left(0, T ; \mathbb{V}^{\prime}\right), \\
\nabla \psi_{n}\left(u^{k^{\prime \prime}}\right) \rightarrow \nabla \bar{\psi}_{n} \text { weakly in } L^{2}(0, T ; \mathbb{H}),
\end{array}\right.
$$

as $k^{\prime \prime} \rightarrow \infty$. Set $\psi_{n}^{k^{\prime \prime}}=\psi_{n}\left(u^{k^{\prime \prime}}\right)$, therefore, for $n$-th particle $P_{n}$, by taking test function $w^{j}=\mathrm{n}^{n}$ in (3), by the definition of weak form (2) to find

$$
\left\{\begin{array}{l}
\int_{0}^{T} \int_{\Omega}-\mathbf{i} \hbar \psi_{n}^{k^{\prime \prime}} \frac{\partial \mathrm{n}^{n}}{\partial t} d \mathbf{x} d t \\
=\int_{0}^{T} \int_{\Omega}-\frac{\hbar^{2}}{2 M} \frac{\partial \psi_{n}^{k^{\prime \prime}}}{\partial \mathbf{x}} \frac{\partial \mathrm{n}^{n}}{\partial \mathbf{x}}+u_{n}(\mathbf{x}) \psi_{n}^{k^{\prime \prime}} \mathrm{n}^{n} j+\mathbf{n} \nu\left|\psi_{n}^{k^{\prime \prime}}\right|^{2} \psi_{n}^{k^{\prime \prime}} \mathrm{n}^{n} d \mathbf{x} d t
\end{array}\right.
$$

If using (5), (6), and taking $k^{\prime \prime} \rightarrow \infty$ to yield that

$$
\left\{\begin{array}{l}
\int_{0}^{T} \int_{\Omega}-\mathbf{i} \hbar \bar{\psi}_{n} \frac{\partial \mathrm{n}^{n}}{\partial t} d \mathbf{x} d t \\
=\int_{0}^{T} \int_{\Omega}-\frac{\hbar^{2}}{2 M} \frac{\partial \bar{\psi}_{n}}{\partial \mathbf{x}} \frac{\partial \mathrm{n}^{n}}{\partial \mathbf{x}}+u_{n}(\mathbf{x}) \bar{\psi}_{n} \mathbf{n}^{n}+\mathbf{n} \nu\left|\bar{\psi}_{n}\right|^{2} \bar{\psi}_{n} \mathrm{n}^{n} d \mathbf{x} d t \quad \forall \mathrm{n}^{n} \in C^{1}(0, T ; \mathbb{V}) .
\end{array}\right.
$$


It inferred that $\bar{\psi}_{n}$ is a solution of (1) in the sense of distribution $\mathcal{D}^{\prime}(0, T)$ on $(0, T)$. From uniqueness of weak solution for BEC system to attain $\bar{\psi}_{n}=\psi_{n}\left(u_{n}^{*}\right)$. For $\psi_{n}$ to get two convergences as

$$
\begin{array}{lll}
\psi_{n}\left(u_{n}^{k^{\prime \prime}}\right) \rightarrow \psi_{n}\left(u_{n}^{*}\right) & \text { strongly in } & L^{2}(0, T ; \mathbb{H}), \\
\psi_{n}^{f}\left(u_{n}^{k^{\prime \prime}}\right) \rightarrow \psi_{n}^{f}\left(u_{n}^{*}\right) & \text { strongly in } \quad \mathbb{H}, \quad k^{\prime \prime} \rightarrow \infty .
\end{array}
$$

Since the norm $\|\cdot\|_{\mathbb{L}^{2}(\Omega)}$ are lower semi-continuous in weak topology of $\mathbb{L}^{2}(\Omega)$, for $n$-th particle $P_{n}$

$$
\liminf _{k^{\prime \prime} \rightarrow \infty}\left\|\psi_{n}^{f}\left(u_{n}^{k^{\prime \prime}}\right)-\psi_{n}^{\text {target }}\right\|_{\mathbb{H}}^{2} \geq\left\|\psi_{n}^{f}\left(u_{n}^{*}\right)-\psi_{n}^{\text {target }}\right\|_{\mathbb{H}}^{2}
$$

Vice versa, from weak convergence (5) that $\lim _{k^{\prime \prime} \rightarrow \infty} \inf \left(u_{n}^{k^{\prime \prime}}, u_{n}^{k^{\prime \prime}}\right) \mathcal{U} \geq\left(u_{n}^{*}, u_{n}^{*}\right) \mathcal{U}$. For cost function (4), $J=\liminf _{k^{\prime \prime} \rightarrow \infty} J\left(u_{n}^{k^{\prime \prime}}\right) \geq J\left(u_{n}^{*}\right)$, and $J\left(u_{n}^{*}\right)=\inf _{u_{n} \in \mathcal{U}_{a d}} J\left(u_{n}\right)$. That is, $u_{n}^{*}$ is quantum optimal control to criteria function (4). That is Theorem 2.

Theorem 3 For $\psi_{n 0} \in \mathbb{V}$, and control problem for $G$-P system (1) to cost function (4), if $\mathcal{U}_{\text {ad }}$ is closed convex (bounded) admissible subset of $\mathcal{U}$, then quantum optimal control $u_{n}^{*}$ is characterized by Eular-Lagrange system as

$$
\begin{aligned}
& \left\{\begin{array}{l}
\mathbf{i} \hbar \frac{\partial \psi_{n}}{\partial t}=-\frac{\hbar^{2}}{2 M} \Delta \psi_{n}+u_{n}^{*}(\mathbf{x}) \psi_{n}+\mathbf{n} \nu\left|\psi_{n}\right|^{2} \psi_{n} \text { in } \mathrm{Q}, \\
\psi_{n}\left(u_{n}^{*}, 0\right)=\psi_{n 0} \text { on } \Omega
\end{array}\right. \\
& \left\{\begin{array}{l}
\mathbf{i} \hbar \frac{\partial \sigma_{n}}{\partial t}=-\frac{\hbar^{2}}{2 M} \Delta \sigma_{n}+2 \mathbf{n} \nu\left|\psi_{n}\right| \psi_{n} \sigma_{n}+\mathbf{n} \nu\left|\psi_{n}\right|^{2} \sigma_{n} \text { in } \mathrm{Q}, \\
\mathbf{i} \sigma_{n}(T)=\psi_{n}^{f}\left(u_{n}^{*}\right)-\psi_{n}^{\text {target }} \text { on } \Omega
\end{array}\right. \\
& \int_{0}^{T} \int_{\Omega} \sigma^{*} \sigma_{n}\left(u_{n}^{*}\right)\left(u-u_{n}^{*}\right) d \mathbf{x} d t+\left(u_{n}^{*}, u_{n}-u_{n}^{*}\right)_{\mathcal{U}} \geq 0 \quad \forall u_{n} \in \mathcal{U}_{a d} .
\end{aligned}
$$

In here, $\sigma_{n} \in W(0, T)$ is solution of adjoint system corresponding to $\psi_{n}$ of BEC system. (7) is necessary optimality condition for quantum optimal control $u_{n}^{*}$ of $n$-th particle $P_{n}$, $n=1,2, \cdots, \mathbf{n} . \sigma^{*}$ is conjugate operator of $\sigma$ in $\sigma\left(\psi_{n}\right)=u_{n}^{*}(\mathbf{x}) \psi_{n}$.

Applying control theory to Bose-Einstein-Condensate is accomplished for the existence of quantum optimal control to Gross-Pitaevskii equation respect to quadratic criteria function. If using practical formulation of electro-magnetic field $v_{n}^{1}(\mathbf{x})$ and optical force $v_{n}^{2}(\mathbf{x})$, control of a condensate can be performed directly and straightforwardly. According to apparatus, detail $v_{n}^{1}(\mathbf{x}), v_{n}^{2}(\mathbf{x})$ can be set up in lab as guidance for controlling particle in physical experiments. For configuration $\left(\sigma_{0}, \mu\right)$ of controls fields $v_{n}^{1}$ and $v_{n}^{2}$ of atomic chips, second order necessary and sufficient equality for criteria function $J\left(u_{n}\right)$ or external force potential $V\left(u_{n}\right)$ at $u_{n}^{*}=\left(v_{n}^{1 *}, v_{n}^{2 *}\right)$ is in variational form as $J^{\prime \prime}\left(u_{n}^{*}\right)=0$ or $\frac{\partial V\left(u_{n}^{*}\right)}{\partial u_{n}^{2}}=-\int_{\Omega} u_{n}^{*} \operatorname{Re}\left[\psi_{n}\left(u_{n}^{*}\right)\right] \operatorname{Re}\left[\sigma_{n}\left(u_{n}^{*}\right)\right] d \mathbf{x}$. Theoretical conclusion would be worked for real-time computer-aided numerical control by adopting of adequate computational algorithm.

In contrast, question from control are left for Bose-Einstein-Condensates. People create condensate of atoms or bosons at low temperature under the magnetic field and 
optical trapping lattice, to make all the particles at the same quantum state. Can BEC condensate be for other purpose at other areas, industrial; aerospace; medical therapy; computer; materials. Condensate out of the door of lab, and into an ideal tools, it is not a dream.

\section{Concludes work}

Quantum control for Bose-Einstein-Condensate is considered for full proof of existence of optimal control in complex Hilbert space. Control theory is applied to BEC in the framework of variational method. It is a confidential theoretical work for complementary of BEC control theory corresponding to computational approach of $[7,8]$.

\section{Acknowledgments}

The author thank to ACS National Meetings \& Exposition, and Cybernetic and Physics.

\section{References}

[1] Dautary R and Lions J L 1992 Mathematical Analysis and Numerical Methods for Science and Technology (Berlin-Heidelberg-New York: Springer-Verlag)

[2] Grond J 2009 Physical Review A $\mathbf{8 0} 053625$

[3] Lions J L 1971 Optimal Control of Systems Governed by Partial Differential Equations (BerlinHeidelberg-New York: Springer-Verlag)

[4] Pitaevskii L P 2003 Internat. Ser. Monogr. Phys. 116 (Oxford UK: Clarendon Press)

[5] Rice S A 2000 Optical Control of Molecular Dynamics (New York: Wiley)

[6] Temam R 1988 Infinite-Dimensional Dynamical Systems in Mechanics and Physis 68 (Applied Math. Sci.: Springer-Verlag)

[7] Wang Q F 2009 International Scientific Conference on Physics and Control Italy

[8] Wang Q F 2013 245-th ACS National Meeting 83 Exposition Poster 'Presentation on Demand'

[9] Wang Q F and Belavkin V P 2012 Cybernetic and Physics 1(2) 144 